\title{
Multi-centre randomised controlled trial comparing arthroscopic hip surgery to physiotherapist-led care for femoroacetabular impingement (FAI) syndrome on hip cartilage metabolism: the Australian FASHIoN trial
}

David J. Hunter ${ }^{1,2^{*}}$, Jillian Eyles ${ }^{1,2}$, Nicholas J. Murphy ${ }^{1,3}$, Libby Spiers ${ }^{4}$, Alexander Burns ${ }^{5}$, Emily Davidson ${ }^{6}$, Edward Dickenson 7,8 , Camdon Fary ${ }^{9,10}$, Nadine E. Foster ${ }^{11,12}$, Jurgen Fripp ${ }^{13}$, Damian R. Griffin ${ }^{7}$, Michelle Hall ${ }^{4}$, Young Jo Kim ${ }^{14}$, James M. Linklater ${ }^{15}$, Robert Molnar ${ }^{16}$, Ales Neubert ${ }^{13}$, Rachel L. O'Connell ${ }^{1,17}$, John O'Donnell ${ }^{18,19}$, Michael O'Sullivan ${ }^{20}$, Sunny Randhawa ${ }^{21}$, Stephan Reichenbach ${ }^{22,23,24}$, Florian Schmaranzer ${ }^{24}$, Parminder Singh ${ }^{18,25}$, Phong Tran ${ }^{9,10}$, David Wilson ${ }^{26}$, Honglin Zhang ${ }^{26}$ and Kim L. Bennell ${ }^{4}$

\begin{abstract}
Background: Arthroscopic surgery for femoroacetabular impingement syndrome (FAl) is known to lead to selfreported symptom improvement. In the context of surgical interventions with known contextual effects and no true sham comparator trials, it is important to ascertain outcomes that are less susceptible to placebo effects. The primary aim of this trial was to determine if study participants with FAI who have hip arthroscopy demonstrate greater improvements in delayed gadolinium-enhanced magnetic resonance imaging (MRI) of cartilage (dGEMRIC) index between baseline and 12 months, compared to participants who undergo physiotherapist-led management.

Methods: Multi-centre, pragmatic, two-arm superiority randomised controlled trial comparing physiotherapist-led management to hip arthroscopy for FAI. FAl participants were recruited from participating orthopaedic surgeons clinics, and randomly allocated to receive either physiotherapist-led conservative care or surgery. The surgical intervention was arthroscopic FAl surgery. The physiotherapist-led conservative management was an individualised physiotherapy program, named Personalised Hip Therapy (PHT). The primary outcome measure was change in dGEMRIC score between baseline and 12 months. Secondary outcomes included a range of patient-reported outcomes and structural measures relevant to FAl pathoanatomy and hip osteoarthritis development. Interventions were compared by intention-to-treat analysis.
\end{abstract}

\footnotetext{
* Correspondence: david.hunter@sydney.edu.au

IInstitute of Bone and Joint Research, Kolling Institute of Medical Research, University of Sydney, Camperdown, Australia

${ }^{2}$ Department of Rheumatology, Royal North Shore Hospital, Sydney, Australia

Full list of author information is available at the end of the article
}

(c) The Author(s). 2021 Open Access This article is licensed under a Creative Commons Attribution 4.0 International License, which permits use, sharing, adaptation, distribution and reproduction in any medium or format, as long as you give appropriate credit to the original author(s) and the source, provide a link to the Creative Commons licence, and indicate if changes were made. The images or other third party material in this article are included in the article's Creative Commons licence, unless indicated otherwise in a credit line to the material. If material is not included in the article's Creative Commons licence and your intended use is not permitted by statutory regulation or exceeds the permitted use, you will need to obtain permission directly from the copyright holder. To view a copy of this licence, visit http://creativecommons.org/licenses/by/4.0/ The Creative Commons Public Domain Dedication waiver (http://creativecommons.org/publicdomain/zero/1.0/) applies to the data made available in this article, unless otherwise stated in a credit line to the data. 
Results: Ninety-nine participants were recruited, of mean age 33 years and 58\% male. Primary outcome data were available for 53 participants (27 in surgical group, 26 in PHT). The adjusted group difference in change at 12 months in dGEMRIC was $-59 \mathrm{~ms}(95 \% \mathrm{Cl}-137.9$ to -19.6$)(p=0.14)$ favouring PHT. Hip-related quality of life (iHOT-33) showed improvements in both groups with the adjusted between-group difference at 12 months showing a statistically and clinically important improvement in arthroscopy of 14 units (95\% Cl 5.6 to 23.9) ( $p=0.003$ ).

Conclusion: The primary outcome of dGEMRIC showed no statistically significant difference between PHT and arthroscopic hip surgery at 12 months of follow-up. Patients treated with surgery reported greater benefits in symptoms at 12 months compared to PHT, but these benefits are not explained by better hip cartilage metabolism.

Trial registration details: Australia New Zealand Clinical Trials Registry reference: ACTRN12615001177549. Trial registered 2/11/2015.

Keywords: Arthroscopy, dGEMRIC, Femoroacetabular impingement syndrome, FAl, Hip, Physiotherapy, Surgery

\section{Background}

Femoroacetabular impingement (FAI) syndrome is a motion-related clinical disorder of the hip with a triad of symptoms, clinical signs, and imaging findings [1]. It is a highly prevalent cause of hip pain in active young adults [2] and considered to be a primary contributor to the development of hip osteoarthritis (OA) [3]. It represents symptomatic premature contact between the proximal femur and acetabulum with two morphologic patterns described: cam and pincer. The repetitive abutment of the proximal femur against the acetabular rim, applying shear forces to the acetabular labrum and/or cartilage, is believed to lead to hip OA [4-6].

Both because of the symptoms associated with FAI and the purported linkages prognostically to hip OA, there is increasing interest in treating the syndrome. Currently, the most popular management approaches include surgery, mainly hip arthroscopy, and physiotherapist-led conservative care [7, 8]. Physiotherapist-led conservative care usually encompasses a multi-faceted approach, including an assessment of the patient's pain, function and hip range of motion; an individualised and progressive exercise program; and education about the condition and its management [7]. Arthroscopic FAI surgery involves resection of the cam and/or pincer morphology, often with surgical repair of concomitant FAI-associated soft tissue pathology, such as acetabular labral tears and chondral defects. Numbers of arthroscopic hip surgeries have been increasing rapidly [8]. Several randomised controlled trials have recently been conducted comparing interventions for FAI [9-13].

The primary outcome for most of these RCTs was self-reported and possibly influenced by placebo effects. In the context of surgical interventions with known contextual effects approaching the minimum clinically important difference [14] and no true sham-comparator trials $[13,15]$, it is important to ascertain outcomes that are less susceptible to placebo effects. Furthermore, given the potential causative role that FAI plays in hip
OA, an important criterion by which each treatment must be evaluated is its effect on the risk of future hip OA. If there are interventions that can not only reduce FAI symptoms but also prevent or slow the onset of OA, it would make a dramatic difference in the interventions recommended for those at risk [1].

A substantial problem in preventive trials in $\mathrm{OA}$ is that meaningful differences between treatment groups can take several decades to occur. It is in this context that this RCT measured several structural outcomes relevant to the pathogenesis of hip OA, with the aim of determining whether hip arthroscopy leads to greater improvements in hip cartilage metabolism than physiotherapist-led conservative care by 12 months follow up and hence differ in their effect on the risk of future hip OA. A prior non-randomized study [16] suggested that joint preserving surgery for FAI was associated with a decline in measures of cartilage metabolism at 1 year but the comparative effects of physiotherapy are unknown.

The primary objective was to compare change in hip cartilage mean dGEMRIC (delayed gadolinium-enhanced MRI of cartilage) score for a region of interest (ROI), including both acetabular and femoral head cartilages at the chondrolabral transitional zone between baseline and 12 months follow-up between the surgery versus conservative care groups. Several secondary objectives were pursued that are detailed in the protocol paper [17].

\section{Methods}

The Australian FASHIoN trial was a pragmatic, assessorand statistician-blinded, two-arm superiority RCT. The Australian FASHIoN [17] (Australian and New Zealand Clinical Trials Registration Number: ACTRN12615001177549, registered 2/11/2015) trial was conducted according to the same protocol for trial design and interventions as the parallel UK FASHIoN trial (Trial Registration number: ISRCTN64081839) [18]. Whereas the primary outcome of the UK FASHIoN trial 
was patient-reported hip-related quality of life (iHOT 33) at 12 months, the primary outcome for this Australian FASHIoN trial was hip cartilage metabolism at 12 months measured using MRI.

The protocol paper provides further detail on the design [17]. This trial was conducted in compliance with the Australian National Health and Medical Research Council (NHMRC) National Statement on Ethical Conduct in Human Research (2007), the Note for Guidance on Good Clinical Practice (CPMP/ICH-135/95), and the conditions of the ethics approval granted by St Vincent's Hospital Human Research Ethics Committee (HREC/14/ $\mathrm{SVH} / 343)$. Results for this trial are reported in accordance with the CONSORT statement (see checklist in Additional file 1: Appendix 1).

\section{Participants and sites}

Participants were recruited through public and private clinics across ten sites in Australia following a diagnosis of FAI by one of eight study orthopaedic surgeons. The personalised hip therapy (PHT) program was administered at 24 private physiotherapy clinics located throughout NSW, Victoria and ACT. Inclusion criteria were: age $\geq 16$ years, hip pain, cam and/or pincer morphology on imaging (alpha angle $>55^{\circ}$ and/or Lateral Centre Edge Angle (LCEA) $>40^{\circ}$ or other radiographic sign of pincer morphology including positive cross-over sign), and the treating surgeon believing the patient would benefit from arthroscopic surgery. Potential participants were excluded if they had evidence of preexisting OA (Tonnis grade $>1$ [19] or $<2 \mathrm{~mm}$ joint space width on pelvic radiograph), previous significant hip pathology, injury, or shape-changing hip surgery.

\section{Randomisation, allocation and blinding}

Randomisation to either arthroscopic hip surgery or physiotherapist-led conservative care occurred in a 1:1 ratio using a computer-generated minimisation sequence (adaptive stratified sampling) with study hospital site and type of FAI (cam, pincer or mixed FAI) as factors [17]. Allocation concealment was preserved by having randomisation codes held by an external biostatistician. At randomisation, participants were given a study ID that was used on all trial documentation.

Neither participants, nor treating surgeons/physiotherapists, could be blinded to treatment allocation. The treating surgeons and physiotherapists took no part in outcome assessment for the trial. Imaging analyses were performed in a blinded fashion. The patient-reported outcome data were collected via online surveys and postal questionnaires and entered onto the central trial database by a research assistant blinded to treatment allocation.

\section{Interventions}

\section{Arthroscopic hip surgery}

Arthroscopic surgery was standardised and performed by one of eight orthopaedic surgeons experienced in arthroscopic hip surgery for FAI [17]. Participants could access surgery through either the public healthcare system, with no out-of-pocket cost, or through the private healthcare system, typically associated with additional out-of-pocket costs, depending on the hospital site from which they were recruited. After giving written informed consent for the procedure, patients underwent routine preoperative care, including an anaesthetic consultation to assess surgical fitness. Surgery was performed under general anaesthesia in either a lateral or supine position. Arthroscopic portals were established in the central and peripheral compartment under radiographic guidance, according to each surgeon's usual practice. Shape abnormalities and consequent labral and cartilage pathologies were treated. Bony resection at the acetabular rim and the head-neck junction were assessed by intraoperative image intensifier radiograph and/or satisfactory impingement free range of movement of the hip. Osteointegrative anchors were used during the surgical procedure to avoid issues with post-operative MRI quality and dGEMRIC accuracy.

Patients were discharged from hospital when they could walk safely with crutches (usually within $24 \mathrm{~h}$ ). A protocol for post-operative rehabilitation was not specified, although patients were instructed to follow the usual post-operative rehabilitation protocol recommended by their surgeon. Physiotherapists providing post-operative rehabilitation care were distinct from those providing the physiotherapy-led conservative care in this trial to avoid contamination between groups.

To assess the fidelity of the treatment received to the prescribed surgical protocol, an international panel of 5 surgeons specialised in hip arthroscopy reviewed all surgical cases. Operation notes, intraoperative images, and postoperative MRI scans were used to evaluate the adequacy of the surgical intervention using techniques previously reported [12].

\section{Physiotherapy-led conservative care}

The physiotherapist-led conservative care was called Personalised Hip Therapy (PHT) [7, 17]. It was designed to represent a consensus on the best conservative care for FAI by an international panel of physiotherapists, physicians, and surgeons. The PHT program was provided at no cost to participants and delivered by experienced musculoskeletal physiotherapists in private practice who were trained in its delivery. Training involved a one-day course explaining the rationale for the FASHIoN trial and the PHT program, and instruction on how to record data related to fidelity of the 
intervention delivery. Participants were offered a minimum of six PHT sessions during the first 12 weeks of the trial, commencing as soon after randomisation as practicable. If needed, participants had additional PHT sessions between 12 weeks and 6 months, up to a total maximum of ten sessions. Further physiotherapy treatment beyond the ten sessions provided by the PHT physiotherapist was not part of the PHT protocol and was recorded as a co-intervention.

The PHT program encompassed a multi-faceted approach, beginning with an assessment of the patient's pain, function and hip range of motion. The core aspects of the program included (i) an individualised and progressive exercise program supervised by a physiotherapist, (ii) education about the condition and its management, and (iii) advice regarding pain relief which could include referral to the participants' General Practitioner, or if necessary referral for an ultrasound-guided intra-articular steroid injection to enable participants to engage in the exercise program where pain would otherwise prevent them from doing so. Physiotherapists were provided with a set of recommended exercises as part of the program, from which they prescribed and progressed appropriate exercises for each participant's stage of rehabilitation. Participants were given a logbook to record the exercises they completed at home. Data from the logbook were not collected by researchers, but were used to enhance physiotherapist-patient communication and facilitate exercise progression and adherence to the program.

The key features of the exercise program were individualisation, progression and supervision; thus, evidence of these features was sought from individual participant PHT case report forms (CRFs). A randomly selected sample of 10 CRFs (at least one from each of the PHT physiotherapists) was assessed by two members of the panel that developed the PHT protocol [7] to assess treatment fidelity and the remaining CRFs by two Australian investigators.

\section{Outcomes}

Participants received an MRI of their hip using standardised sequences [17] at baseline and 12 months on one of three $3 \mathrm{~T}$ scanners (Siemens Prisma, Siemens Skyra or Phillips Ingenia). Because of its proven reliability, the dGEMRIC technique was used for quantification and detection of changes in the glycosaminoglycan (GAG) content of the hip joint cartilage [20-23]. To this end, participants received an intravenous injection of the contrast agent; $0.2 \mathrm{mmol} / \mathrm{kg}$ bodyweight of Dotarem (GdDOTA; Guerbet, Cedex, France) or Magnevist (GdDTPA; Berlex Labs, Wayne, NJ). Following the injection of the contrast agent, participants walked for $15 \mathrm{~min}$, after which MRI scanning occurred and dGEMRIC sequences were acquired 45-60 min post-injection. The primary outcome measure for analysis was the betweengroup difference in change from baseline to 12 months in the average $\mathrm{T}_{1}$ relaxation time, assessed with dGEMRIC, for a region of interest (ROI) comprising both acetabular and femoral head cartilages at the chondrolabral transitional zone of the mid-sagittal plane. The betweengroup difference in the change from baseline to 12months in separate acetabular and femoral head cartilage ROIs at the chondrolabral transitional zones were analysed as a secondary outcome. In addition, the 12month change in standardised dGEMRIC $\mathrm{z}$-scores for the acetabular and femoral head cartilage ROIs at the chondrolabral junction were also assessed as secondary outcomes [24, 25].

Secondary outcomes included hip joint structural change between baseline and 12 months as demonstrated by the semi-quantitative whole hip OA MRI Score (HOAMS), a validated and reliable semiquantitative whole hip OA MRI scoring system [26]. Participants also received standardised plain radiographs, comprising supine anteroposterior (AP) pelvis, false profile, and 45-degree modified Dunn views. Plain radiographic (measured using Hip2Norm [27]) and MRI measures of alpha angle, acetabular depth, femoral (baseline only) and acetabular version were reported at baseline and 12 months.

A range of other secondary outcomes were collected including change in: hip related quality of life as measured by the international Hip Outcome Tool-33 (iHOT-33) [28], the Hip disability and Osteoarthritis Outcome Score (HOOS) [29-31] EQ-5D [32], and the 12-Item Short Form Health Survey (SF-12) [33]; patientperceived overall improvement following intervention measured using a Global Improvement Scale (GIS); and patient satisfaction with care and treatment results measured on a five-point Likert scale. Participants completed the Modified UCLA (University of California Los Angeles) activity score [34]. Age of symptom onset was calculated as age of presentation minus reported duration of symptoms. Procedures for measurement and analysis of each of the secondary outcomes are described in greater detail in the protocol paper [17]. The number and type of adverse events was recorded for all participants up to 12 months. For further details, see the study flow diagram (Fig. 1).

\section{Statistical analysis}

The primary intention to treat analysis was the change in the dGEMRIC index score for a combined acetabular and femoral head cartilage ROI from baseline to 12 months, with the difference in mean change between the two intervention groups presented with a 95\% confidence interval and compared using an independent 


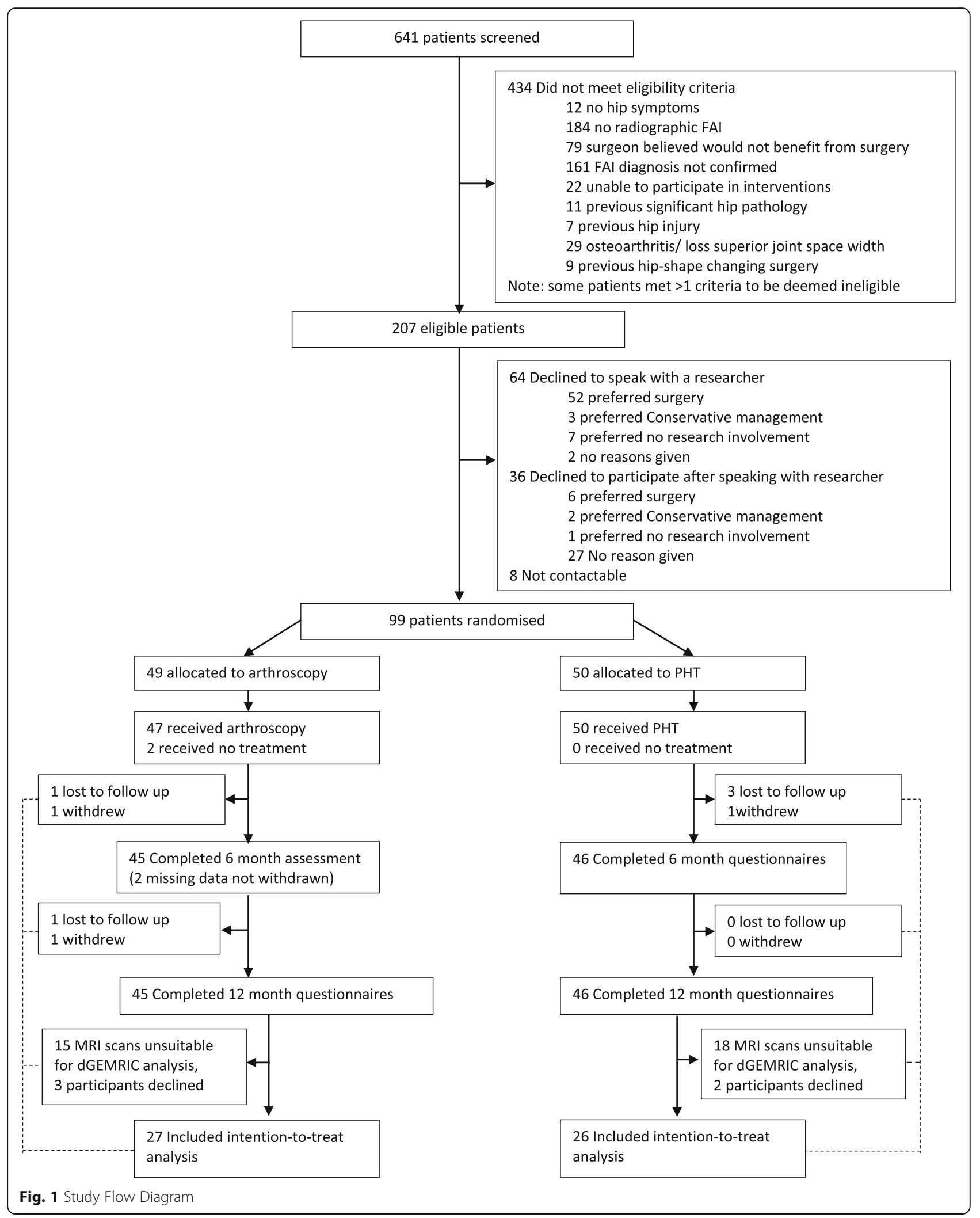


samples t-test [17]. This strategy also applied for the standardised dGEMRIC z-scores. Analysis including adjustment for baseline dGEMRIC index score and relevant baseline characteristics performed using Analysis of Covariance with the change from baseline modelled as the dependent variable. This strategy was also applied for the dGEMRIC index score of the separate acetabular and femoral head cartilage ROIs and the standardized dGEMRIC z-scores.

This strategy was also used for analysis of other approximately normally distributed secondary outcome measures, including those related to plain radiography, MRI, and the patient reported outcomes (iHOT-33, HOOS, EQ-5D and SF-12). Differences in dichotomous outcome variables such as adverse events, complications related to the trial interventions and the need for further procedures were compared between groups using chisquared tests (or Fisher's exact test). Although our inferences are drawn from the intention-to-treat analysis, we also performed a pre-specified per-protocol analyses. We performed pre-specified exploratory subgroup analyses by FAI type and by whether referral to the trial was via the private or public healthcare systems. If participant adherence to, or completion of the PHT proved to be more problematic than expected, we intended to augment the planned analysis with a complier average causal effect (CACE) analysis.

Economic and biomechanical analyses will be reported in a subsequent publication.

\section{Sample size}

Sample size calculations for the primary outcome were based on a statistical power of $90 \%$, and two-sided significance of $5 \%$ significance level. With a sample size of 54 patients in each group, we expected to detect a difference of at least $50 \mathrm{~ms}$ between two intervention groups at 12 months based on a standard deviation (SD) of 80 $\mathrm{ms}$ for the dGEMRIC index [22, 35]. $50 \mathrm{~ms}$ was chosen as clinically significant based upon an increased risk of subsequent total hip replacement [36]. We aimed to recruit a total of 140 participants to allow for a drop-out rate of $20 \%$, including $5 \%$ cross-over to the surgical group. (See Additional file 1: Appendix 3 for details on reduced sample size, which came about in large part as a consequence of changes in Medicare funding for hp. arthroscopy shortly after the study commenced).

\section{Results}

Ninety-nine participants (of whom we have primary outcome data on 53-see Flow Chart (Fig. 1)) were recruited from February 17, 2015 to December 30th 2017, of which 49 were allocated to arthroscopy, 50 allocated to PHT and 3 patients crossed over from PHT to arthroscopy during the 12 months of the study. Our participants had a mean age of 33 years, were predominantly male (58\%), 20\% had bilateral symptoms, the median duration of symptoms was 20 months, and cam type FAI was predominant (63\%). More details on the participants are included in Table 1 . The average time to commencement of treatment after randomisation was 12.5 (SD (8.1) weeks for arthroscopy and 4.7 (SD 2.3) weeks for PHT. Those with complete dGEMRIC data were broadly similar to those that had missing dGEMRIC data (Supplementary Table 8). The between group change in dGEMRIC is outlined in Table 2 . The adjusted group difference in the primary dGEMRIC combined outcomes was $-59 \mathrm{~ms}(95 \% \mathrm{CI}-137.9$ to -19.6$)(p=$ 0.14), the direction of effect favouring PHT in terms of hip cartilage metabolism but was not statistically significant. Between group effects for acetabular and femoral ROI were similar, with more acetabular change and again non-significant. A per-protocol analysis $(n=39)$ found similar results (Supplementary Table 1).

A range of secondary outcomes are presented in Table 3. iHOT-33 showed improvements in both groups with the adjusted group difference showing a statistically and clinically important greater improvement in the arthroscopy group of 14.2 units ( $95 \%$ CI 5.6 to 23.9 ) ( $p=$ $0.003)$. There were similarly greater improvements in quality of life (EQ-5D-5L), HOOS pain and symptoms in the arthroscopy group compared to the PHT group. There were statistically significant between group differences in the maximum MRI alpha angle $(p=0.001)$, along with radiographic between group differences in LCEA and extrusion index favouring arthroscopy.

Results of the semi-quantitative MRI analysis (HOAMS) are presented in Table 4. These demonstrate worsening in cartilage score $(p=0.002)$, an increase in number of regions worsened for cartilage score $(p=$ 0.0002 ), and an increase in the number of worsened regions for labral score $(p=0.0009)$ for arthroscopy compared to PHT. In contrast, there was a reduction in the number of regions affected by osteophytes $(p=0.01)$ for hip arthroscopy compared to PHT. No participants had loose bodies, attrition or dysplasia. Baseline HOAMS scores are included in Supplementary Table 9.

Per protocol dGEMRIC analysis is broadly consistent with the results presented in Table 2 (Supplementary Table 1). Subgroup analyses comparing FAI type (cam vs mixed vs pincer) (Supplemental Table 2), age (Supplementary Table 3) and public vs private study hospital sites (Supplementary Table 5) did not demonstrate any significant between group differences. Participants with a baseline dGEMRIC index above the median (indicating better cartilage metabolism) demonstrated a significant between group difference in change of $-110.7 \mathrm{~ms}$, favouring PHT $(p=0.035)$ (Supplementary Table 4). 
Table 1 Baseline Characteristics ( $N=99)$

\begin{tabular}{|c|c|c|c|c|}
\hline Characteristic & & $\begin{array}{l}\text { Arthroscopy } \\
N=49\end{array}$ & $\begin{array}{l}P H T \\
N=50\end{array}$ & $\begin{array}{l}\text { Total } \\
N=99\end{array}$ \\
\hline Age (years, mean [SD]) & & $32.9(11.8)$ & $32.9(9.1)$ & $32.9(10.5)$ \\
\hline \multirow[t]{2}{*}{ Gender: } & Male & $31(63 \%)$ & $26(52 \%)$ & $57(58 \%)$ \\
\hline & Female & $18(37 \%)$ & $24(48 \%)$ & $42(42 \%)$ \\
\hline \multirow[t]{3}{*}{ Current smoker: } & Yes & $5(10 \%)$ & $5(10 \%)$ & $10(10 \%)$ \\
\hline & No & 41 (84\%) & $44(88 \%)$ & $85(86 \%)$ \\
\hline & Missing & $3(6 \%)$ & $1(2 \%)$ & $4(4 \%)$ \\
\hline \multirow[t]{2}{*}{ Hip side to be considered for treatment: } & right & $22(45 \%)$ & $24(48 \%)$ & $46(46 \%)$ \\
\hline & left & $27(55 \%)$ & $26(52 \%)$ & $53(54 \%)$ \\
\hline \multirow[t]{2}{*}{ Bilateral symptoms: } & Yes & $9(18 \%)$ & $11(22 \%)$ & $20(20 \%)$ \\
\hline & No & $40(82 \%)$ & $39(78 \%)$ & 79 (80\%) \\
\hline Duration of hip symptoms (mths, median [min, max]) & & $24.0(2.0,84.0)$ & $18.0(2.5,120.0)$ & $20.0(2.0,120.0)$ \\
\hline \multirow[t]{3}{*}{ Type of FAl: } & Pincer & $9(18 \%)$ & $9(18 \%)$ & $18(18 \%)$ \\
\hline & Mixed & $10(20 \%)$ & $9(18 \%)$ & $19(19 \%)$ \\
\hline & Cam & $30(61 \%)$ & $32(64 \%)$ & $62(63 \%)$ \\
\hline Units of alcohol in average week (median [min, max]) & & $3.0(0.0,16.0)$ & $2.0(0.0,12.0)$ & $2.0(0.0,16.0)$ \\
\hline \multirow[t]{3}{*}{ Diabetic: } & Yes & $1(2 \%)$ & & $1(1 \%)$ \\
\hline & No & 46 (94\%) & $49(98 \%)$ & $95(96 \%)$ \\
\hline & Missing & $2(4 \%)$ & $1(2 \%)$ & $3(3 \%)$ \\
\hline \multirow[t]{2}{*}{ Diagnosed chronic renal failure: } & No & 47 (96\%) & $49(98 \%)$ & $96(97 \%)$ \\
\hline & Missing & $2(4 \%)$ & $1(2 \%)$ & $3(3 \%)$ \\
\hline \multicolumn{2}{|l|}{ Physical activity (UCLA score, mean [SD]) } & $7.5(2.6)$ & $7.2(2.8)$ & $7.3(2.7)$ \\
\hline \multicolumn{2}{|l|}{ HOOS pain (mean [SD]) } & $59.4(18.4)$ & $57.4(18.9)$ & $58.4(18.6)$ \\
\hline \multicolumn{2}{|l|}{ HOOS Symptom (mean [SD]) } & $49.7(17.5)$ & $50.7(20.9)$ & $50.2(19.2)$ \\
\hline \multicolumn{2}{|l|}{ HOOS ADL (mean [SD]) } & $69.2(18.0)$ & $65.9(20.4)$ & $67.5(19.3)$ \\
\hline \multicolumn{2}{|l|}{ HOOS Sport \& Recreation (mean [SD]) } & $47.9(22.4)$ & $46.9(23.6)$ & $47.4(22.9)$ \\
\hline \multicolumn{2}{|l|}{ HOOS Quality of Life (mean [SD]) } & $33.6(16.1)$ & $30.1(17.6)$ & $31.8(16.9)$ \\
\hline \multicolumn{2}{|l|}{ Hip related Quality of Life (i-HOT-33, (mean [SD])) } & $43.5(17.6)$ & $40.4(20.0)$ & $41.9(18.8)$ \\
\hline \multicolumn{2}{|l|}{ SF-12 PCS (mean [SD]) } & $40(7.8)$ & $39(8.4)$ & $39(8.1)$ \\
\hline \multicolumn{2}{|l|}{ SF-12 MCS (mean [SD]) } & $49(11.2)$ & $48(10.8)$ & $49(10.9)$ \\
\hline \multicolumn{2}{|l|}{ EQ-5D-5L index score (mean [SD]) } & $0.575(0.21)$ & $0.544(0.23)$ & $0.559(0.22)$ \\
\hline \multicolumn{2}{|l|}{ EQ-5D-5L VAS (mean [SD]) } & $68.9(16.3)$ & $68.8(14.2)$ & $68.8(15.2)$ \\
\hline \multicolumn{2}{|l|}{ Maximum MRI alpha angle (mean [SD]) } & $70.2(11.9)$ & $70.6(15.6)$ & $70.4(13.9)$ \\
\hline \multicolumn{2}{|l|}{ dGEMRIC Combined (mean [SD]) } & $679.6(118.6)$ & $667.0(127.4)$ & $673.6(121.8)$ \\
\hline \multicolumn{2}{|l|}{ dGEMRIC Acetabular ROI (mean [SD]) } & $661.2(121.1)$ & $637.2(145.3)$ & $649.7(132.4)$ \\
\hline \multicolumn{2}{|l|}{ dGEMRIC Femoral ROI (mean [SD]) } & $698.9(137.0)$ & $698.4(125.4)$ & $698.7(130.2)$ \\
\hline \multicolumn{2}{|l|}{ dGEMRIC Z-score (mean [SD]) } & $-0.49(0.70)$ & $-0.45(0.67)$ & $-0.47(0.68)$ \\
\hline \multicolumn{2}{|l|}{ Total AP anterior coverage (\%, mean [SD]) } & $27.2(8.2)$ & $24.7(6.2)$ & $25.9(7.3)$ \\
\hline \multicolumn{2}{|l|}{ Total AP posterior coverage $(\%$, mean $[S D])$} & $47.4(8.1)$ & $45.0(8.3)$ & $46.2(8.3)$ \\
\hline Total Femur coverage $(\%$, mean $[\mathrm{SD}])$ & & $82.4(7.6)$ & $81.0(7.3)$ & $81.7(7.4)$ \\
\hline LCE $(\%$, mean $[S D])$ & & $37.1(5.4)$ & $34.7(6.6)$ & $35.8(6.2)$ \\
\hline LCE & $<25$ & & $2(4 \%)$ & $2(2 \%)$ \\
\hline & $25+$ & 47 (96\%) & $48(96 \%)$ & $95(96 \%)$ \\
\hline & Missing & $2(4 \%)$ & & $2(2 \%)$ \\
\hline
\end{tabular}


Table 1 Baseline Characteristics ( $N=99)$ (Continued)

\begin{tabular}{|c|c|c|c|c|}
\hline Characteristic & & $\begin{array}{l}\text { Arthroscopy } \\
N=49\end{array}$ & $\begin{array}{l}P H T \\
N=50\end{array}$ & $\begin{array}{l}\text { Total } \\
N=99\end{array}$ \\
\hline \multicolumn{5}{|l|}{ Radiographic Measurements } \\
\hline Acetabular Index (\%, mean [SD]) & & $2.5(4.2)$ & $4.7(4.8)$ & $3.6(4.6)$ \\
\hline ACM angle $(\%$, mean $[S D])$ & & $45.0(2.5)$ & $44.7(3.6)$ & $44.8(3.1)$ \\
\hline Extrusion Index (\%, mean [SD]) & & $15.0(4.5)$ & $17.0(5.7)$ & $16.0(5.3)$ \\
\hline \multirow[t]{3}{*}{ Cross-over-sign } & Yes & $31(63 \%)$ & $38(76 \%)$ & $69(70 \%)$ \\
\hline & No & $16(33 \%)$ & $12(24 \%)$ & $28(28 \%)$ \\
\hline & Missing & $2(4 \%)$ & & $2(2 \%)$ \\
\hline Retroversion Index (\%, median [min, max]) & & $6.7(0.0,46.0)$ & $11.1(0.0,54.7)$ & $10.2(0.0,54.7)$ \\
\hline \multirow[t]{3}{*}{ Posterior wall sign } & Yes & $29(59 \%)$ & $37(74 \%)$ & $66(67 \%)$ \\
\hline & No & $18(37 \%)$ & $13(26 \%)$ & $31(31 \%)$ \\
\hline & Missing & $2(4 \%)$ & & $2(2 \%)$ \\
\hline
\end{tabular}

Data are number (\%) unless otherwise indicated. UCLA University of California Los Angeles. iHOT-33 International Hip Outcome Tool. SF-12 12-item Short Form Health Survey. PCS Physical component score. MCS Mental component score

LCEA Lateral centre edge angle. Hip Osteoarthritis MRI Scoring System (HOAMS). ROI = region of interest

ACM Angle constructed by the following points: $(\mathrm{A})$ lateral edge of the acetabulum, $(\mathrm{M})$ midpoint of a line connecting the lateral and the inferior acetabular edge, (C) point of the bony acetabulum intersecting the perpendicular line relative to line AM through point $M$ Hip2Norm results are for study hip

Table 2 Primary outcome of hip cartilage metabolism assessed by dGEMRIC (ms): Change from Baseline (B) to 12 month (12 M) assessments $(N=53)$

\begin{tabular}{|c|c|c|c|c|c|c|c|c|}
\hline \multirow[t]{2}{*}{ Outcome } & \multicolumn{2}{|c|}{ Arthroscopy $(N=27)$} & \multicolumn{2}{|c|}{$P H T(N=26)$} & \multicolumn{4}{|c|}{ Arthroscopy - PHT } \\
\hline & $\bar{n}$ & $\begin{array}{l}\text { Mean (SD or } 95 \% \\
\text { Cl) }\end{array}$ & $n$ & $\begin{array}{l}\text { Mean (SD or } 95 \% \\
\text { Cl) }\end{array}$ & $\begin{array}{l}\text { Unadjusted } \\
\text { difference }\end{array}$ & $\begin{array}{l}P- \\
\text { value }^{\mathrm{a}}\end{array}$ & $\begin{array}{l}\text { Adjusted difference }(95 \% \\
\text { Cl) }\end{array}$ & $\begin{array}{l}P- \\
\text { value }^{\mathrm{b}}\end{array}$ \\
\hline \multicolumn{9}{|c|}{ dGEMRIC Combined (primary outcome) } \\
\hline Baseline & 26 & $679.6(118.6)$ & 24 & $667.0(127.4)$ & & & & \\
\hline 12 month & 21 & $677.0(122.8)$ & 23 & $722.8(145.7)$ & & & & \\
\hline $\begin{array}{l}\text { Change: } 12 \mathrm{M}- \\
\mathrm{B}\end{array}$ & 20 & $-14.8(-73.8-44.2)$ & 21 & $48.4(-16.6-113.5)$ & -63.2 & 0.142 & $-59.1(-137.9-19.6)$ & 0.137 \\
\hline \multicolumn{9}{|c|}{ dGEMRIC Acetabular ROI } \\
\hline Baseline & 26 & $661.2(121.1)$ & 24 & $637.2(145.3)$ & & & & \\
\hline 12 month & 21 & $618.5(130.5)$ & 23 & $651.8(145.0)$ & & & & \\
\hline $\begin{array}{l}\text { Change: } 12 \mathrm{M}- \\
\text { B }\end{array}$ & 20 & $-63.0(-120.2--5.9)$ & 21 & $7.1(-55.5-69.7)$ & -70.1 & 0.093 & $-58.9(-134.8-17.1)$ & 0.125 \\
\hline \multicolumn{9}{|c|}{ dGEMRIC Femoral ROI } \\
\hline Baseline & 26 & $698.9(137.0)$ & 24 & $698.4(125.4)$ & & & & \\
\hline 12 month & 21 & $735.8(135.6)$ & 23 & $789.8(170.8)$ & & & & \\
\hline $\begin{array}{l}\text { Change: } 12 \mathrm{M}- \\
\text { B }\end{array}$ & 20 & $34.1(-37.6-105.7)$ & 21 & $84.2(8.9-159.5)$ & -50.1 & 0.321 & $-55.4(-149.5-38.6)$ & 0.240 \\
\hline \multicolumn{9}{|l|}{ dGEMRIC Z-score } \\
\hline Baseline & 26 & $-0.49(0.70)$ & 24 & $-0.45(0.67)$ & & & & \\
\hline 12 month & 21 & $-0.50(0.51)$ & 23 & $-0.69(0.54)$ & & & & \\
\hline $\begin{array}{l}\text { Change: } 12 \mathrm{M}- \\
\text { B }\end{array}$ & 20 & $0.16(-0.21-0.53)$ & 21 & $-0.20(-0.63-0.23)$ & 0.36 & 0.199 & $0.24(-0.11-0.58)$ & 0.169 \\
\hline
\end{tabular}

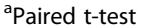

${ }^{\mathrm{b}}$ Based on regression model including adjustment for baseline 
Table 3 Secondary outcomes: Change from Baseline (B) to 6 month (6 M) and 12 month (12 M) assessments (N=99

\begin{tabular}{|c|c|c|c|c|c|c|c|c|}
\hline \multirow[t]{2}{*}{ Outcome } & \multicolumn{2}{|c|}{$\begin{array}{l}\text { Arthroscopy } \\
N=49\end{array}$} & \multicolumn{2}{|c|}{$\begin{array}{l}P H T \\
N=50\end{array}$} & \multicolumn{4}{|c|}{ Arthroscopy - PHT } \\
\hline & $\bar{n}$ & $\begin{array}{l}\text { Mean (SD or } 95 \% \\
C I)\end{array}$ & $\bar{n}$ & $\begin{array}{l}\text { Mean (SD or } 95 \% \\
C I)\end{array}$ & $\begin{array}{l}\text { Unadjusted } \\
\text { difference }\end{array}$ & $\begin{array}{l}P- \\
\text { value }^{a}\end{array}$ & $\begin{array}{l}\text { Adjusted difference (95\% } \\
\text { CI) }\end{array}$ & $\begin{array}{l}P \text { - } \\
\text { value }^{c}\end{array}$ \\
\hline \multicolumn{9}{|l|}{ iHOT-33 } \\
\hline Baseline & 48 & 43.5 (17.6) & 50 & $40.4(20.0)$ & & & & \\
\hline 6 month & 44 & $62.2(21.2)$ & 42 & $57.5(24.9)$ & & & & \\
\hline Change: $6 \mathrm{M}-\mathrm{B}$ & 44 & $18.6(13.0-24.2)$ & 42 & $13.3(7.2-19.3)$ & 5.4 & 0.194 & $5.2(-2.7-13.2)$ & 0.196 \\
\hline 12 month & 45 & $72.9(21.8)$ & 46 & $56.8(28.8)$ & & & & \\
\hline Change: $12 \mathrm{M}-\mathrm{B}$ & 45 & $29.6(22.9-36.3)$ & 46 & $15.4(8.8-22.1)$ & 14.2 & 0.003 & $14.7(5.6-23.9)$ & 0.002 \\
\hline \multicolumn{9}{|l|}{ EQ-5D-5L index score } \\
\hline Baseline & 48 & $0.575(0.206)$ & 50 & $0.544(0.231)$ & & & & \\
\hline 6 month & 44 & $0.703(0.177)$ & 41 & $0.682(0.155)$ & & & & \\
\hline Change: $6 \mathrm{M}-\mathrm{B}$ & 44 & $0.121(0.068-0.174)$ & 41 & $0.089(0.033-0.145)$ & 0.032 & 0.405 & $0.026(-0.035-0.087)$ & 0.398 \\
\hline 12 month & 45 & $0.770(0.156)$ & 46 & $0.653(0.249)$ & & & & \\
\hline Change: $12 \mathrm{M}-\mathrm{B}$ & 45 & $0.194(0.131-0.256)$ & 46 & $0.101(0.033-0.169)$ & 0.093 & 0.046 & $0.106(0.029-0.183)$ & 0.007 \\
\hline \multicolumn{9}{|l|}{ EQ5D-VAS } \\
\hline Baseline & 48 & $68.9(16.3)$ & 50 & $68.8(14.2)$ & & & & \\
\hline 6 month & 45 & $72.7(16.8)$ & 42 & $71.7(16.1)$ & & & & \\
\hline Change: $6 \mathrm{M}-\mathrm{B}$ & 45 & $3.7(-1.5-9.0)$ & 42 & $1.3(-4.4-7.0)$ & 2.4 & 0.530 & $1.6(-4.9-8.1)$ & 0.627 \\
\hline 12 month & 45 & 76.9 (15.2) & 46 & 74.7 (14.6) & & & & \\
\hline Change: $12 \mathrm{M}-\mathrm{B}$ & 45 & $7.9(2.4-13.3)$ & 46 & $5.8(1.0-10.6)$ & 2.1 & 0.570 & $2.1(-3.7-7.9)$ & 0.470 \\
\hline \multicolumn{9}{|l|}{ HOOS Pain } \\
\hline Baseline & 47 & $59.4(18.4)$ & 50 & $57.4(18.9)$ & & & & \\
\hline 6 month & 45 & $74.0(17.8)$ & 45 & $67.6(23.5)$ & & & & \\
\hline Change: $6 \mathrm{M}-\mathrm{B}$ & 43 & $13.2(7.0-19.4)$ & 45 & $10.9(5.7-16.1)$ & 2.3 & 0.567 & $3.7(-3.8-11.2)$ & 0.327 \\
\hline 12 month & 41 & 83.9 (16.0) & 42 & 71.3 (19.9) & & & & \\
\hline Change: $12 \mathrm{M}-\mathrm{B}$ & 41 & $24.8(17.7-32.0)$ & 42 & $11.1(5.2-17.1)$ & 13.7 & 0.004 & $12.9(5.4-20.5)$ & 0.001 \\
\hline \multicolumn{9}{|l|}{ HOOS Symptom } \\
\hline Baseline & 47 & 49.7 (17.5) & 50 & $50.7(20.9)$ & & & & \\
\hline 6 month & 45 & $64.4(19.7)$ & 45 & $59.8(25.6)$ & & & & \\
\hline Change: $6 \mathrm{M}-\mathrm{B}$ & 43 & $14.4(8.3-20.5)$ & 45 & $8.7(3.5-13.8)$ & 5.8 & 0.149 & $5.3(-2.3-12.9)$ & 0.168 \\
\hline 12 month & 41 & 73.8 (18.8) & 42 & $63.8(22.8)$ & & & & \\
\hline Change: $12 \mathrm{M}-\mathrm{B}$ & 41 & $23.2(16.1-30.3)$ & 42 & $9.6(3.4-15.8)$ & 13.5 & 0.005 & $11.7(3.3-20.1)$ & 0.007 \\
\hline \multicolumn{9}{|l|}{ HOOS ADL } \\
\hline Baseline & 47 & $69.2(18.0)$ & 50 & $65.9(20.4)$ & & & & \\
\hline 6 month & 45 & 80.8 (18.2) & 45 & $75.0(24.0)$ & & & & \\
\hline Change: $6 \mathrm{M}-\mathrm{B}$ & 43 & $11.1(5.1-17.2)$ & 45 & $9.2(4.7-13.6)$ & 2.0 & 0.591 & $3.1(-3.9-10.1)$ & 0.377 \\
\hline 12 month & 41 & 89.7 (14.3) & 42 & $76.6(21.4)$ & & & & \\
\hline Change: $12 \mathrm{M}-\mathrm{B}$ & 41 & $20.4(14.6-26.2)$ & 42 & $7.4(2.4-12.3)$ & 13.0 & 0.001 & $13.0(6.4-19.7)$ & 0.000 \\
\hline \multicolumn{9}{|c|}{ HOOS Sport and Recreation } \\
\hline Baseline & 47 & $47.9(22.4)$ & 50 & $46.9(23.6)$ & & & & \\
\hline 6 month & 45 & $61.1(19.9)$ & 45 & $58.5(27.9)$ & & & & \\
\hline Change: $6 \mathrm{M}-\mathrm{B}$ & 43 & $12.8(6.0-19.6)$ & 45 & $12.5(5.9-19.1)$ & 0.3 & 0.951 & $1.1(-7.4-9.6)$ & 0.803 \\
\hline 12 month & 41 & $75.2(17.5)$ & 42 & $62.5(25.4)$ & & & & \\
\hline Change: $12 \mathrm{M}-\mathrm{B}$ & 41 & $28.4(20.7-36.0)$ & 42 & $13.2(5.9-20.6)$ & 15.1 & 0.005 & $13.7(4.9-22.4)$ & 0.003 \\
\hline
\end{tabular}


Table 3 Secondary outcomes: Change from Baseline (B) to 6 month $(6 \mathrm{M})$ and 12 month $(12 \mathrm{M})$ assessments ( $N=99$ (Continued)

\begin{tabular}{|c|c|c|c|c|c|c|c|c|}
\hline \multirow[t]{2}{*}{ Outcome } & \multicolumn{2}{|c|}{$\begin{array}{l}\text { Arthroscopy } \\
N=49\end{array}$} & \multicolumn{2}{|c|}{$\begin{array}{l}P H T \\
N=50\end{array}$} & \multicolumn{4}{|c|}{ Arthroscopy - PHT } \\
\hline & $n$ & $\begin{array}{l}\text { Mean (SD or } 95 \% \\
\text { Cl) }\end{array}$ & $n$ & $\begin{array}{l}\text { Mean (SD or } 95 \% \\
\text { Cl) }\end{array}$ & $\begin{array}{l}\text { Unadjusted } \\
\text { difference }\end{array}$ & $\begin{array}{l}P- \\
\text { value }^{a}\end{array}$ & $\begin{array}{l}\text { Adjusted difference (95\% } \\
\text { CI) }\end{array}$ & $\begin{array}{l}P \text { - } \\
\text { value }\end{array}$ \\
\hline \multicolumn{9}{|l|}{ Retroversion Index } \\
\hline Baseline & 47 & $11.3(12.8)$ & 50 & $14.7(14.4)$ & & & & \\
\hline 12 month & 42 & $9.3(10.7)$ & 43 & $11.3(14.3)$ & & & & \\
\hline Change: $12 \mathrm{M}-\mathrm{B}$ & 42 & $-0.8(-3.9-2.2)$ & 43 & $-2.5(-6.7-1.7)$ & 1.6 & 0.527 & $0.0(-4.6-4.6)$ & 0.997 \\
\hline
\end{tabular}

iHOT-33 International Hip; Hip Osteoarthritis MRI Scoring System (HOAMS)

${ }^{\text {aP }}$ aired t-test

bilcoxon rank-sum test

${ }^{\mathrm{c}}$ Based on regression model including adjustment for baseline

Surgery for the majority of surgical participants demonstrated satisfactory fidelity (84\%) but was deemed inadequate for seven participants (16\%) (Supplementary Table 6). PHT was also judged as satisfactory in terms of intervention fidelity for the majority of participants $(82 \%$ of PHT participants).

PHT was generally well tolerated with 25 participants (53\%) complaining of muscle soreness from exercise. In the hip arthroscopy surgery group, 33\% of participants complained of numbness in the groin, leg or foot; and $31 \%$ had some problems as a consequence of taking pain medication (Supplementary Table 7).

\section{Discussion}

The primary outcome of hip cartilage metabolism dGEMRIC showed no statistically significant difference between PHT and arthroscopic hip surgery at 12 months follow-up. Since the loss of GAG in cartilage is an early OA-related change [37], dGEMRIC enables the likelihood of future OA development to be compared between interventions after a relatively short period [35]. It is important to be cognisant that a range of issues led to a reduction in the required sample size and there is a trend in cartilage metabolism favouring PHT. The baseline standard deviation in our trial participants $(\sim 120$ $\mathrm{ms}$ ) is greater than the $80 \mathrm{~ms}$ used in our sample size calculation, suggesting heterogeneity of this measure in our trial population which would have further underpowered our ability to detect a significant difference and raising the distinct possibility that the lack of statistical significance observed is a Type 2 error. The mean difference in the arthroscopic treatment group was small and the between group difference was comparable with the estimate that we used for the sample size calculation. The results of semi-quantitative MRI analyses demonstrated worse cartilage and labral scores in the arthroscopic group at 12 months.

Some potential reasons for the findings relate to MRI cartilage metabolism. During hip arthroscopy, surgeons often perform an acetabular chondroplasty / microfracture / or chondral repair. Furthermore, during a labral repair the procedure involves separating any chondro-labral adhesions or scar with a rasp prior to repair. All of these procedures could adversely affect labral and cartilage scores. Another potential mechanism is that surgery introduces a transient inflammatory state that may adversely affect cartilage biochemical content in the short to medium term. The decline that we have seen in dGEMRIC indices has been found in prior investigations and the magnitude of the decline found in our surgical group is broadly consistent with prior studies [16]. Further, there may be positive effects of exercise on glycosaminoglycan content consistent with what we have found in our PHT group [38, 39].

Several randomised controlled trials have recently been conducted comparing interventions for FAI [9-13]. Potentially as a consequence of their differing designs, including a variety of comparator interventions, study settings, and eligibility criteria; the RCTs to date show both surgery and conservative care can lead to average benefits overall, but have had inconsistent conclusions regarding superiority of one versus another. In our trial, from a patient-reported symptom standpoint, the range of secondary outcomes demonstrated statistically and clinically important improvements with significant between group differences favouring surgery [14] in IHOT-33, quality of life, HOOS pain and symptoms at 12 months, similar to the UK FASHIoN trial [12]. Intriguingly, while trending in the same direction, there were no significant differences in improvements between groups at 6 months. Both groups demonstrated symptom improvements at 12 months, but the arthroscopic group consistently demonstrated greater benefit. The magnitude of effect and separation from PHT comparator is broadly consistent with prior randomised controlled trials $[12,40]$. The results of semi-quantitative MRI analyses demonstrated worse cartilage and labral scores in the arthroscopic group at 12 months. In addition, participants with a baseline dGEMRIC above the median, indicating better cartilage metabolism, also 
Table 4 HOAMS (semi-quantitative MRI) outcomes: Changes over 12 months. Values are number (\%), unless otherwise indicated

\begin{tabular}{|c|c|c|c|c|}
\hline MRI feature/category & $\begin{array}{l}\text { Arthroscopy } \\
N=42\end{array}$ & $\begin{array}{l}\mathrm{PHT} \\
N=43\end{array}$ & Difference, $\%(95 \% \mathrm{Cl})$ & $P$-value \\
\hline \multicolumn{5}{|l|}{ Cartilage } \\
\hline \multicolumn{5}{|l|}{ Change across all regions (excluding within-grade change): } \\
\hline No change & $29(69 \%)$ & $43(100 \%)$ & & $0.0002^{\mathrm{a}}$ \\
\hline Worsening in 1 subregion & $11(26 \%)$ & 0 & & \\
\hline Worsening in 2 subregions & $2(5 \%)$ & 0 & & \\
\hline Any worsening & $13(31)$ & 0 & $31(15-47)$ & \\
\hline \multicolumn{5}{|l|}{ Change across all regions (including within-grade change): } \\
\hline Improvement in 1 subregion+ worsening in 1 subregion & $1(2 \%)$ & 0 & & \\
\hline No change & $26(62 \%)$ & $41(95 \%)$ & & \\
\hline Worsening in 1 subregion only & $11(26 \%)$ & $2(5 \%)$ & & \\
\hline Worsening in 2 subregions only & $3(7 \%)$ & 0 & & \\
\hline Worsening in $3+$ subregions only & $1(2 \%)$ & 0 & & \\
\hline Any worsening & $16(38 \%)$ & $2(5 \%)$ & $33(15-52)$ & $0.0002^{\mathrm{b}}$ \\
\hline \multicolumn{5}{|l|}{ BML } \\
\hline \multicolumn{5}{|l|}{ Change in no. of subregions affected by any lesion: } \\
\hline Improvement & $1(2 \%)$ & $1(2 \%)$ & & $0.61^{\mathrm{a}}$ \\
\hline No change & $38(91 \%)$ & $40(93 \%)$ & & \\
\hline Worsening in 1 subregion & $1(2 \%)$ & $2(5 \%)$ & & \\
\hline Worsening in 2 subregions & $2(5 \%)$ & 0 & & \\
\hline \multicolumn{5}{|l|}{ Maximum change in BML score across all subregions: } \\
\hline No change ${ }^{c}$ & $37(88 \%)$ & $40(93 \%)$ & & $0.66^{\mathrm{a}}$ \\
\hline Within-grade worsening & $2(5 \%)$ & 0 & & \\
\hline Worsening by 1 grade & $3(7 \%)$ & $3(7 \%)$ & & \\
\hline Any subregion with improvement (including within-grade changes) in BML & $2(5 \%)$ & $4(9 \%)$ & $-4(-18-9)$ & \\
\hline Any subregion with worsening (including within-grade changes) in BML & $5(12 \%)$ & $3(7 \%)$ & $5(-10-20)$ & \\
\hline \multicolumn{5}{|l|}{ Subchondral cyst } \\
\hline \multicolumn{5}{|l|}{ Change in no. of subregions affected: } \\
\hline Improvement & $5(12 \%)$ & 0 & & $0.11^{\mathrm{a}}$ \\
\hline No change & $33(78 \%)$ & $39(91 \%)$ & & \\
\hline Worsening in 1 subregion & $4(10 \%)$ & $3(7 \%)$ & & \\
\hline Worsening in 2 subregions & 0 & 0 & & \\
\hline Worsening in 3 subregions & 0 & $1(2 \%)$ & & \\
\hline \multicolumn{5}{|l|}{ Maximum change in score across all subregions: } \\
\hline No change ${ }^{c}$ & $38(90 \%)$ & $37(86 \%)$ & & $0.75^{\mathrm{a}}$ \\
\hline Within-grade worsening & 0 & $2(5 \%)$ & & \\
\hline Worsening by 1 grade & $4(10 \%)$ & $4(9 \%)$ & & \\
\hline Any subregion with improvement (including within-grade changes) & $7(17 \%)$ & $3(7 \%)$ & $10(-6-26)$ & \\
\hline Any subregion with worsening (including within-grade changes) & $4(10 \%)$ & $6(14 \%)$ & $-4(-20-12)$ & \\
\hline \multicolumn{5}{|l|}{ Osteophyte } \\
\hline Increase in no. locations affected by any osteophyte: & 0 & 0 & & \\
\hline \multicolumn{5}{|l|}{ Change in no. of locations affected by any osteophyte: } \\
\hline Improvement 1 subregion & $6(14 \%)$ & 0 & & $0.01^{b}$ \\
\hline No Change & $36(86 \%)$ & $42(100 \%)$ & & \\
\hline
\end{tabular}


Table 4 HOAMS (semi-quantitative MRI) outcomes: Changes over 12 months. Values are number (\%), unless otherwise indicated (Continued)

\begin{tabular}{|c|c|c|c|c|}
\hline MRI feature/category & $\begin{array}{l}\text { Arthroscopy } \\
N=42\end{array}$ & $\begin{array}{l}\text { PHT } \\
N=43\end{array}$ & Difference, \% (95\% Cl) & $P$-value \\
\hline Maximum change in osteophyte score $\geq 1$ across all subregions: & 0 & 0 & & \\
\hline \multicolumn{5}{|l|}{ Labrum } \\
\hline \multicolumn{5}{|l|}{ Maximum change in score across all subregions: } \\
\hline No change ${ }^{c}$ & $17(40 \%)$ & $34(81 \%)$ & & \multirow[t]{6}{*}{$0.0009^{a}$} \\
\hline Within-grade worsening & $12(29 \%)$ & $3(7 \%)$ & & \\
\hline Worsening by 1 grade & $11(26 \%)$ & $5(12 \%)$ & & \\
\hline Worsening by 2 grades & $2(5 \%)$ & 0 & & \\
\hline Any subregion with improvement (including within-grade changes) & $7(17 \%)$ & $2(5 \%)$ & $12(-3-27)$ & \\
\hline Any subregion with worsening (including within-grade changes) & $25(60 \%)$ & $8(19 \%)$ & $40(19-62)$ & \\
\hline \multicolumn{5}{|l|}{ Synovitis } \\
\hline \multicolumn{5}{|l|}{ Maximum change in score across all subregions: } \\
\hline No change ${ }^{c}$ & $14(33 \%)$ & $23(58 \%)$ & & \multirow[t]{6}{*}{$0.08^{\mathrm{a}}$} \\
\hline Within-grade worsening & $7(17 \%)$ & $5(12 \%)$ & & \\
\hline Worsening by 1 grade & $19(45 \%)$ & $11(28 \%)$ & & \\
\hline Worsening by 2 grades & $2(5 \%)$ & $1(2 \%)$ & & \\
\hline Any subregion with improvement (including within-grade changes) & $10(24 \%)$ & $10(24 \%)$ & $-0(-21-20)$ & \\
\hline Any subregion with worsening (including within-grade changes) & $28(67 \%)$ & $17(41 \%)$ & $25(2-48)$ & \\
\hline
\end{tabular}

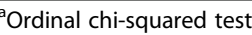

${ }^{b}$ Chi-squared test

Includes within-grade improvement

demonstrated a clear separation in between group dGEMRIC difference favouring the PHT arm. The magnitude of that difference $(111 \mathrm{~ms})$ is what would be considered clinically meaningful, as a threshold of $50 \mathrm{~ms}$ has prognostic value for an increased risk of subsequent total hip replacement in hip dysplasia [36]. Several studies on hip dysplasia and FAI have shown that dGEMRIC as a metric can detect cartilage changes in early OA, and predict the outcomes of joint preserving surgery, as well as the likelihood of surgical failure and requirement for total hip replacement $[35,36,41,42]$. In contrast, semiquantitative MRI scores for osteophytes improved in the surgical arm, but this may reflect their resection during the arthroscopic procedure.

In this regard, further assessment of the longer term clinical outcomes is critical. This will be forthcoming for these trial participants and in addition analyses focused on identifying whether there is any association between structural changes found on the MRI at 12 months and the longer term (3-5 years) self-reported symptom outcome measures will be critical in understanding the prognostic value of these cartilage metabolism findings.

There are a number of strengths of our trial that warrant mentioning. The design was rigorous and the outcomes are reliable $[12,17]$. The demographics of our trial population are broadly consistent with prior studies, in particular the UK FASHIoN trial [12, 40]. In addition, there are a number of limitations. Unfortunately, we were not able to recruit the targeted sample size in large part because of a change in funding for hip arthroscopy by the Australian government that impacted our recruitment. In addition, a number of concerns around gadolinium based contrast agents and potential deposition in the brain [43] led to a change from Magnevist to Dotarem. In addition, the currently reported followup duration is short at only 12 months.

The need for RCTs to determine the most effective approach for the management of FAI is well-recognised [44]. While this trial suggests short-term (12 months) changes in cartilage metabolism, the longer term prognostic significance of these as it relates to clinical outcomes remains unknown. The data should be available within the next 2 to 3 years. Furthermore, the biomechanical effects of surgery and their prognostic value also will be determined as will health economic analyses. Further research, ideally with a credible sham surgery comparator is needed to identify whether the superiority of surgery at 12 months in these RCTs is explained by nonspecific effects [45].

\section{Conclusions}

In conclusion, the primary outcome of dGEMRIC showed no statistically significant difference between arthroscopic hip surgery and conservative care (PHT) at 
12 months of follow-up, although this may represent a type 2 error. Patients treated with surgery reported greater improvements in symptoms at 12 months compared to PHT. Both interventions provide benefits, on average, for patients but surgery more so than PHT. This trial adds new information that shows the patient reported benefits of surgery are not explained by nor linked to better hip cartilage metabolism at 12 months.

\section{Supplementary Information}

The online version contains supplementary material available at https://doi. org/10.1186/s12891-021-04576-z.

Additional file 1: Appendix 1. CONSORT Checklist. Appendix 2. MRI missing data. Appendix 3. Details on rationale for reduced sample size. Appendix 4. Statistical analysis plan. Appendix 5. Protocol.

Additional file 2: Supplementary Table 1. FASHION Per-protocol analysis: dGEMRIC (ms): Change from Baseline (B) and 12 month (12M) assessments ( $N=39$ ). Supplementary Table 2. dGEMRIC (ms) Combined: Change from Baseline (B) and 12 month (12M) assessments ( $N=53)$. Subgroup analysis FAI type. Supplementary Table 3. FASHION: dGEMRIC (ms) Combined: Change from Baseline (B) and 12 month (12M) assessments $(N=53)$. Supplementary Table 4. FASHION: dGEMRIC (ms) Combined: Change from Baseline (B) and 12 month (12M) assessments ( $N=$ 50). Supplementary Table 5. dGEMRIC Combined (ms): Change from Baseline (B) and 12 month (12M) assessments ( $N=53)$. Subgroup analysis: Public vs Private. Supplementary Table 6. Intervention Fidelity. Supplementary Table 7. All patient-reported adverse events $n(\%)$. Supplementary Table 8. FASHION: Comparison of Baseline Characteristics for patients with dGEMRIC data vs missing ( $N=99)$. Supplementary Table 9. Baseline MRI HOAMS features. Numbers are $\mathrm{n}(\%)$.

\section{Acknowledgements}

We acknowledge the participants and staff who contributed their time and efforts to data collection. UK FASHIoN, from which the protocol was derived, was funded by the National Institute for Health Research in the UK [12]. We would like to thank the following radiology clinic staff for their support for this project: Dr. Peter Smith from I-MED Radiology Network, VIC, Sunita Alwa and Brad Shiell from Castlereagh Radiology, St Leonards, NSW. We would also like to thank the following physiotherapists who delivered the PHT intervention in NSW, Victoria and ACT: Nicole Baer, Active Care Physiotherapy \& Sports Therapy, Bondi Junction, NSW; Emma Gillingham, Your Health Domain, Sydney, NSW, Brendan Limbrey, Arrow Physiotherapy, Castle Hill, NSW, Warren Ansell, Proactive Physiotherapy, Tamworth, NSW; Matt Becker, Coffs Coast Sports Physiotherapy, Coffs Harbour, NSW; Dave Hillard, Zone 34, Lewisham, NSW; Drew Renkert, Military Road Physiotherapy, Neutral Bay, NSW; Steve Davey, Mosman Physiotherapy, Mosman, NSW; Brooke Cranney, Dynamic Performance Physiotherapy, Gymea, NSW; David Pattison, NSW; April-Rose Ferris, NSW; Ben Curtis, ACT; Tony Beecroft, Melbourne Sports Physiotherapy, Essendon, VIC; Andrew Dalwood, Physioworks Camberwell, Camberwell, VIC; Daniel Yee, West Active, Werribee, VIC; Laura Parlby, LifeCare, Croydon, VIC; Chris Snell, VIC; Amy Cusworth, LifeCare Croydon, VIC; Daniel Zwolak, Physica, Ringwood, VIC; Melissa Allen, VIC, Wade Byrnes, Waurn Ponds Physiotherapy Clinic, Waurn Ponds, VIC.

\section{Patient and public involvement}

Our protocol was informed by the UK FASHloN feasibility study conducted in the UK https://www.journalslibrary.nihr.ac.uk/hta/hta20320/\#/abstract. This mixed methods study explored consumer willingness to participate, and informed the development of trial procedures, including an optimal recruitment consultation which we used in our trial. Building on this initial work, we invited two consumers to provide feedback over the design and protocol for the Australian arm of the study, particularly the radiological protocols, burden of intervention and time required to participate in the research. They were not involved in plans to disseminate the study results.

\section{Authors' contributions}

DJH, NEF, DRG, YJK, JML, JOD, and KLB contributed to study conception and design. $D J H, J O D$ and KLB attained project funding. DJH drafted the first version of the manuscript. ROC performed the statistical analysis. All authors revised the manuscript critically for important intellectual content and read and approved the final version. All authors agree to be accountable for all aspects of the work.

\section{Funding}

This study was funded by a National Health and Medical Research Council of Australia grant (APP1069278) and by the Australian Hip Arthroscopy Education and Research Foundation.

\section{Availability of data and materials}

Data are available upon reasonable request

Deidentified participant data are available from the corresponding author for collaboration. Additional information including the protocol [17] and statistical analysis plan is also available.

\section{Declarations}

\section{Ethics approval and consent to participate}

This protocol has been approved by St Vincent's Hospital Human Research Ethics Committee (HREC/14/SVH/343). Informed consent was obtained from all study participants before participation in the study. All methods were carried out in accordance with relevant guidelines and regulations.

\section{Consent for publication}

Not applicable.

\section{Competing interests}

DJH is a consultant to Pfizer, Lilly, TLCBio and Merck Serono and is supported by an NHMRC Practitioner Fellowship. KLB was supported by an NHMRC Principal Research Fellowship (APP1058440). SMG acknowledges the support of the Parker-Hughes endowment and the Frecker Family bequest. NEF is an NIHR Senior Investigator in the UK and was supported through an NIHR Research Professorship (NIHR-RP-011-015). MH was supported by a Sir Randal Heymanson Research Fellowship from The University of Melbourne. The rest of the authors do not have any competing interest.

\section{Author details}

${ }^{1}$ Institute of Bone and Joint Research, Kolling Institute of Medical Research, University of Sydney, Camperdown, Australia. 'Department of Rheumatology, Royal North Shore Hospital, Sydney, Australia. ${ }^{3}$ Department of Orthopaedic Surgery, Gosford and Wyong Hospitals, Gosford, New South Wales, Australia. ${ }^{4}$ Department of Physiotherapy, Centre for Health, Exercise and Sports Medicine, University of Melbourne, Parkville, Australia. ${ }^{5}$ Orthopaedics ACT, 90 Corinna St, Canberra 2603, Australia. ${ }^{6}$ Department of Radiology, Royal Prince Alfred Hospital, Sydney, New South Wales 2035, Australia. ${ }^{7}$ Warwick Medical School, University of Warwick, Coventry, UK. ${ }^{8}$ University Hospitals of Coventry and Warwickshire NHS Trust, Coventry, UK. ${ }^{9}$ Department of Orthopaedic Surgery, Western Health, Melbourne, Australia. ${ }^{10}$ Australian Institute for Musculoskeletal Science (AIMSS), The University of Melbourne and Western Health, St. Albans, VIC, Australia. "'STARS Education and Research Alliance, School of Health and Rehabilitation Sciences, University of Queensland, St Lucia, Australia. ${ }^{12}$ Primary Care Centre Versus Arthritis, School of Medicine, Keele University, Newcastle upon Tyne, UK. ${ }^{13}$ The Australian e-Health Research Centre, CSIRO Health and Biosecurity, Brisbane, Australia. ${ }^{14}$ Department of Orthopedic Surgery, Boston Children's Hospital, 300 Longwood Avenue, Boston, MA 02115, USA. ${ }^{15}$ Department of Musculoskeletal Imaging, Castlereagh Imaging, St Leonards, New South Wales, Australia. ${ }^{16}$ Sydney Orthopaedic Trauma \& Reconstructive Surgery, Sydney, New South Wales, Australia. ${ }^{17}$ NHMRC Clinical Trials Centre, University of Sydney, Camperdown, Australia. ${ }^{18} \mathrm{Hip}$ Arthroscopy Australia, 21 Erin St, Richmond, Victoria, Australia. ${ }^{19}$ St Vincent's Private Hospital, 159 Grey St, East Melbourne, Victoria, Australia. ${ }^{20}$ North Sydney Orthopaedic and Sports Medicine Centre, North Sydney, New South Wales, Australia. ${ }^{21}$ Macquarie University Hospital, 3 Technology PI, Macquarie University, Macquarie Park, NSW 2109, Australia. ${ }^{22}$ Institute of Social and Preventive Medicine, University of Bern, Bern, Switzerland. ${ }^{23}$ Department of Rheumatology, Immunology and Allergology, University Hospital and 
University of Bern, Bern, Switzerland. ${ }^{24}$ Department Diagnostic, Interventional and Paediatric Radiology, Inselspital, Bern University Hospital, University of Bern, Bern, Switzerland. ${ }^{25}$ Maroondah Hospital, Eastern Health, Davey Drive, Ringwood East, Melbourne, Victoria 3135, Australia. ${ }^{26}$ Department of Orthopaedics, Center for Hip Health and Mobility, University of British Columbia, Vancouver, BC, Canada.

Received: 2 June 2021 Accepted: 4 August 2021

Published online: 16 August 2021

\section{References}

1. Griffin DR, Dickenson EJ, Agricola R, Awan T, Beck M, Dijkstra P, et al. The 2016 Warwick agreement on Femoroacetabular impingement. Br J Sports Med. 2016;50(19):1162-3.

2. Ganz R, Parvizi J, Beck M, Leunig M, Notzli H, Siebenrock KA. Femoroacetabular impingement: a cause for osteoarthritis of the hip. Clin Orthop Relat Res. 2003;417:112-20.

3. Agricola R, Waarsing JH, Arden NK, Carr AJ, Bierma-Zeinstra SM, Thomas GE, et al. Cam impingement of the hip: a risk factor for hip osteoarthritis. Nat Rev Rheumatol. 2013;9(10):630-4. https://doi.org/10.1038/nrrheum.2013.114.

4. Ganz R, Leunig M, Leunig-Ganz K, Harris WH. The etiology of osteoarthritis of the hip: an integrated mechanical concept. Clin Orthop Relat Res. 2008; 466(2):264-72. https://doi.org/10.1007/s11999-007-0060-z.

5. Beck M, Kalhor M, Leunig M, Ganz R. Hip morphology influences the pattern of damage to the acetabular cartilage: femoroacetabular impingement as a cause of early osteoarthritis of the hip. J Bone Joint Surg Br. 2005;87(7): 1012-8.

6. Ganz R, Gill TJ, Gautier E, Ganz K, Krugel N, Berlemann U. Surgical dislocation of the adult hip a technique with full access to the femoral head and acetabulum without the risk of avascular necrosis. J Bone Joint Surg Br. 2001;83(8):1119-24. https://doi.org/10.1302/0301-620X.83B8.083111 9.

7. Wall PD, Dickenson EJ, Robinson D, Hughes I, Realpe A, Hobson R, et al. Personalised hip therapy: development of a non-operative protocol to treat femoroacetabular impingement syndrome in the FASHIoN randomised controlled trial. Br J Sports Med. 2016;50(19):1217-23. https://doi.org/10.113 6/bjsports-2016-096368.

8. Montgomery SR, Ngo SS, Hobson T, Nguyen S, Alluri R, Wang JC, et al. Trends and demographics in hip arthroscopy in the United States. Arthroscopy. 2013;29(4):661-5. https://doi.org/10.1016/j.arthro.2012.11.005.

9. Krych AJ, Thompson M, Knutson Z, Scoon J, Coleman SH. Arthroscopic labral repair versus selective labral debridement in female patients with femoroacetabular impingement: a prospective randomized study. Arthroscopy. 2013;29(1):46-53. https://doi.org/10.1016/j.arthro.2012.07.011.

10. Mansell NS, Rhon DI, Meyer J, Slevin JM, Marchant BG. Arthroscopic surgery or physical therapy for patients with Femoroacetabular impingement syndrome: a randomized controlled trial with 2-year follow-up. Am J Sports Med. 2018;46(6):1306-14. https://doi.org/10.1177/0363546517751912.

11. Palmer AJR, Ayyar Gupta V, Fernquest S, Rombach I, Dutton SJ, Mansour R, et al. Arthroscopic hip surgery compared with physiotherapy and activity modification for the treatment of symptomatic femoroacetabular impingement: multicentre randomised controlled trial. BMJ. 2019;364:1185.

12. Griffin DR, Dickenson EJ, Wall PDH, Achana F, Donovan JL, Griffin J, et al. Hip arthroscopy versus best conservative care for the treatment of femoroacetabular impingement syndrome (UK FASHION): a multicentre randomised controlled trial. Lancet. 2018;391(10136):2225-35. https://doi. org/10.1016/S0140-6736(18)31202-9

13. Femoroacetabular Impingement Randomized Controlled Trial I, Ayeni OR, Karlsson J, Heels-Ansdell D, Thabane L, Musahl V, et al. Osteochondroplasty and Labral Repair for the Treatment of Young Adults With Femoroacetabular Impingement: A Randomized Controlled Trial. Am J Sports Med. 2021;49(1):25-34

14. Levy DM, Kuhns BD, Chahal J, Philippon MJ, Kelly BT, Nho SJ. Hip arthroscopy outcomes with respect to patient acceptable symptomatic state and minimal clinically important difference. Arthroscopy. 2016;32(9): 1877-86. https://doi.org/10.1016/j.arthro.2016.05.014

15. Louw A, Diener I, Fernandez-de-Las-Penas C, Puentedura EJ. Sham surgery in orthopedics: a systematic review of the literature. Pain Med. 2017;18(4): 736-50. https://doi.org/10.1093/pm/pnw164.

16. Schmaranzer F, Haefeli PC, Hanke MS, Liechti EF, Werlen SF, Siebenrock KA et al. How does the dGEMRIC index change after surgical treatment for FAl?
A prospective controlled study: preliminary results. Clin Orthop Relat Res. 2017:475(4):1080-99. https://doi.org/10.1007/s11999-016-5098-3.

17. Murphy NJ, Eyles J, Bennell KL, Bohensky M, Burns A, Callaghan FM, et al. Protocol for a multi-Centre randomised controlled trial comparing arthroscopic hip surgery to physiotherapy-led care for femoroacetabular impingement (FAl): the Australian FASHIoN trial. BMC Musculoskelet Disord. 2017;18(1):406. https://doi.org/10.1186/s12891-017-1767-y.

18. Griffin DR, Dickenson EJ, Wall PD, Donovan JL, Foster NE, Hutchinson CE, et al. Protocol for a multicentre, parallel-arm, 12-month, randomised, controlled trial of arthroscopic surgery versus conservative care for femoroacetabular impingement syndrome (FASHIoN). BMJ Open. 2016;6(8): e012453. https://doi.org/10.1136/bmjopen-2016-012453.

19. Tonnis D, Heinecke A. Acetabular and femoral anteversion: relationship with osteoarthritis of the hip. J Bone Joint Surg Am. 1999;81(12):1747-70. https:// doi.org/10.2106/00004623-199912000-00014.

20. Zilkens C, Tiderius CJ, Krauspe R, Bittersohl B. Current knowledge and importance of dGEMRIC techniques in diagnosis of hip joint diseases. Skelet Radiol. 2015;44(8):1073-83. https://doi.org/10.1007/s00256-015-2135-3.

21. Mamisch TC, Kain MS, Bittersohl B, Apprich S, Werlen S, Beck M, et al. Delayed gadolinium-enhanced magnetic resonance imaging of cartilage (dGEMRIC) in Femoacetabular impingement. J Orthop Res. 2011;29(9):130511. https://doi.org/10.1002/jor.21371.

22. Domayer SE, Mamisch TC, Kress I, Chan J, Kim YJ. Radial dGEMRIC in developmental dysplasia of the hip and in femoroacetabular impingement: preliminary results. Osteoarthritis Cartilage. 2010;18(11):1421-8. https://doi. org/10.1016/j.joca.2010.08.010.

23. Bittersohl B, Hosalkar HS, Haamberg T, Kim YJ, Werlen S, Siebenrock KA, et al. Reproducibility of dGEMRIC in assessment of hip joint cartilage: a prospective study. J Magn Reson Imaging. 2009;30(1):224-8. https://doi. org/10.1002/jmri.21822.

24. Lattanzi R, Petchprapa C, Glaser C, Dunham K, Mikheev AV, Krigel A, et al. A new method to analyze dGEMRIC measurements in femoroacetabular impingement: preliminary validation against arthroscopic findings. Osteoarthr Cartil. 2012;20(10):1127-33. https://doi.org/10.1016/j.joca.2012.06. 012.

25. Lattanzi R, Petchprapa C, Ascani D, Babb JS, Chu D, Davidovitch Rl, et al, Detection of cartilage damage in femoroacetabular impingement with standardized dGEMRIC at 3 T. Osteoarthr Cartil. 2014;22(3):447-56. https:// doi.org/10.1016/j.joca.2013.12.022.

26. Roemer FW, Hunter DJ, Winterstein A, Li L, Kim YJ, Cibere J, et al. Hip osteoarthritis MRI scoring system (HOAMS): reliability and associations with radiographic and clinical findings. Osteoarthr Cartil. 2011;19(8):946-62. https://doi.org/10.1016/j.joca.2011.04.003.

27. Tannast M, Mistry S, Steppacher SD, Reichenbach S, Langlotz F, Siebenrock KA, et al. Radiographic analysis of femoroacetabular impingement with Hip2Norm-reliable and validated. J Orthop Res. 2008;26(9):1199-205. https:// doi.org/10.1002/jor.20653.

28. Mohtadi NG, Griffin DR, Pedersen ME, Chan D, Safran MR, Parsons N, et al. The Development and validation of a self-administered quality-of-life outcome measure for young, active patients with symptomatic hip disease: the International Hip Outcome Tool (iHOT-33). Arthroscopy. 2012;28(5):595605 quiz 6-10 e1.

29. HOOS User's Guide 2003 [Available from: www.koos.nu/HOOSGuide2003. pdf.

30. Kemp JL, Collins NJ, Roos EM, Crossley KM. Psychometric properties of patient-reported outcome measures for hip arthroscopic surgery. Am J Sports Med. 2013;41(9):2065-73. https://doi.org/10.1177/03635465134 94173.

31. Hinman RS, Dobson F, Takla A, O'Donnell J, Bennell KL. Which is the most useful patient-reported outcome in femoroacetabular impingement? Testretest reliability of six questionnaires. Br J Sports Med. 2014;48(6):458-63. https://doi.org/10.1136/bjsports-2012-092072.

32. Walters SJ, Brazier JE. Comparison of the minimally important difference for two health state utility measures: EQ-5D and SF-6D. Qual Life Res. 2005; 14(6):1523-32. https://doi.org/10.1007/s11136-004-7713-0.

33. Ostendorf M, van Stel HF, Buskens E, Schrijvers AJ, Marting LN, Verbout AJ, et al. Patient-reported outcome in total hip replacement. A comparison of five instruments of health status. J Bone Joint Surg Br. 2004;86(6):801-8.

34. Zahiri CA, Schmalzried TP, Szuszczewicz ES, Amstutz HC. Assessing activity in joint replacement patients. J Arthroplast. 1998;13(8):890-5. https://doi. org/10.1016/50883-5403(98)90195-4. 
35. Kim YJ, Jaramillo D, Millis MB, Gray ML, Burstein D. Assessment of early osteoarthritis in hip dysplasia with delayed gadolinium-enhanced magnetic resonance imaging of cartilage. J Bone Joint Surg Am. 2003;85-A(10):1987-92.

36. Cunningham T, Jessel R, Zurakowski D, Millis MB, Kim YJ. Delayed gadolinium-enhanced magnetic resonance imaging of cartilage to predict early failure of Bernese periacetabular osteotomy for hip dysplasia. J Bone Joint Surg Am. 2006;88(7):1540-8. https://doi.org/10.2106/00004623-2 00607000-00015.

37. Bashir A, Gray ML, Hartke J, Burstein D. Nondestructive imaging of human cartilage glycosaminoglycan concentration by MRI. Magn Reson Med. 1999; 41(5):857-65. https://doi.org/10.1002/(SICl)1522-2594(199905)41:5<857::AIDMRM1 >3.0.CO;2-E.

38. Van Ginckel A, Baelde N, Almqvist KF, Roosen P, McNair P, Witvrouw E. Functional adaptation of knee cartilage in asymptomatic female novice runners compared to sedentary controls. A longitudinal analysis using delayed gadolinium enhanced magnetic resonance imaging of cartilage (dGEMRIC). Osteoarthr Cartil. 2010;18(12):1564-9. https://doi.org/10.1016/j. joca.2010.10.007.

39. Roos EM, Dahlberg L, Roos EM, Dahlberg L. Positive effects of moderate exercise on glycosaminoglycan content in knee cartilage: a four-month, randomized, controlled trial in patients at risk of osteoarthritis. Arthritis Rheum. 2005;52(11):3507-14. https://doi.org/10.1002/art.21415.

40. Kierkegaard S, Langeskov-Christensen M, Lund B, Naal FD, Mechlenburg I, Dalgas $U$, et al. Pain, activities of daily living and sport function at different time points after hip arthroscopy in patients with femoroacetabular impingement: a systematic review with meta-analysis. Br J Sports Med 2017;51 (7):572-9. https://doi.org/10.1136/bjsports-2016-096618.

41. Jessel RH, Zurakowski D, Zilkens C, Burstein D, Gray ML, Kim YJ. Radiographic and patient factors associated with pre-radiographic osteoarthritis in hip dysplasia. J Bone Joint Surg Am. 2009;91(5):1120-9. https://doi.org/10.2106/JBJS.G.00144.

42. Jessel RH, Zilkens C, Tiderius C, Dudda M, Mamisch TC, Kim YJ. Assessment of osteoarthritis in hips with femoroacetabular impingement using delayed gadolinium enhanced MRI of cartilage. J Magn Reson Imaging. 2009;30(5): 1110-5. https://doi.org/10.1002/jmri.21830

43. Olchowy C, Cebulski K, Lasecki M, Chaber R, Olchowy A, Kalwak K, et al. The presence of the gadolinium-based contrast agent depositions in the brain and symptoms of gadolinium neurotoxicity - a systematic review. PLoS One. 2017:12(2):e0171704. https://doi.org/10.1371/journal.pone.0171704.

44. Wall PD, Brown JS, Parsons N, Buchbinder R, Costa ML, Griffin D. Surgery for treating hip impingement (femoroacetabular impingement). Cochrane Database Syst Rev. 2014;9:CD010796.

45. Zou K, Wong J, Abdullah N, Chen X, Smith T, Doherty M, et al. Examination of overall treatment effect and the proportion attributable to contextual effect in osteoarthritis: meta-analysis of randomised controlled trials. Ann Rheum Dis. 2016;75(11):1964-70. https://doi.org/10.1136/annrheumdis-201 5-208387.

\section{Publisher's Note}

Springer Nature remains neutral with regard to jurisdictional claims in published maps and institutional affiliations.

Ready to submit your research? Choose BMC and benefit from:

- fast, convenient online submission

- thorough peer review by experienced researchers in your field

- rapid publication on acceptance

- support for research data, including large and complex data types

- gold Open Access which fosters wider collaboration and increased citations

- maximum visibility for your research: over $100 \mathrm{M}$ website views per year

At BMC, research is always in progress.

Learn more biomedcentral.com/submissions 